\title{
Paradigmas tecnológicos musicais face às diferentes eras da racionalidade humana
}

\author{
Correlations between the evolution of human rationality and music \\ technology paradigms
}

Tatiana Catanzaro

Universidade de Brasília

Resumo: A partir da constatação de que a racionalidade musical em particular e a humana em geral é condicionada, ao mesmo tempo que a influencia de forma dialética, pela evolução tecnológica de cada período histórico, confrontamos duas diferentes classificações concernindo racionalidade e tecnologia propostas pelo musicólogo francês François Delalande e pelo filósofo francês Pierre Lévy. Tecemos considerações sobre as razões que levam ambos os pesquisadores a chegar a diferentes conclusões concernindo as mudanças paradigmáticas da tecnologia no século $X X$, analisando o desenvolvimento histórico da música nos períodos moderno e contemporâneo. Essa metodologia nos permite, por conseguinte, erigir os pilares que permitiram a instauração da "música baseada no som" e de definir as particularidades dessa nova gramatologia extraída do cerne da própria matéria sonora.

Palavras-chave: Tecnologia musical, racionalidade humana, música eletroacústica, música computacional, escrita baseada no som.

\begin{abstract}
Musical (in particular) and human (in general) rationality is conditioned by the technological evolution of each historical period, at the same time as it exerts a reciprocal influence in a dialectical way. Based on these observations, we confront two different classifications concerning rationality and technology proposed by the French musicologist François Delalande and the French philosopher Pierre Lévy. We make considerations about the reasons that lead both researchers to reach different conclusions concerning the paradigmatic changes of technology in the twentieth century, analyzing the historical development of music in the modern and contemporary periods. This methodology allows us, therefore, to erect the pillars that allowed the establishment of a "sound-based music" and to define the particularities of this new grammatology extracted from the very core of sound matter itself.
\end{abstract}

Keywords: Musical technology, human rationality, electroacoustic music, computational music, sound-based writing. 
O essencial não está em adicionar ao seu pensamento uma dimensão tecnológica, mas em discernir o impacto que essa dimensão pode ter sobre o pensamento ${ }^{1}$

(Gérard Grisey)

Como defendido no livro publicado em 2018, Transformações na linguagem musical contemporânea instrumental e vocal sob a influência da música eletroacústica entre as décadas de 1950-70 (p. 13ss), a racionalidade musical em particular e a humana em geral é condicionada, ao mesmo tempo que a influencia de forma dialética, pela evolução tecnológica de cada período histórico. No entanto, apesar dessa influência ser constante, é importante ressaltar como algumas tecnologias acabaram por abalar de maneira mais consequente o intelecto humano.

A pesquisadora em Ciência da Computação e da Comunicação Marie-Noëlle Heinrich e o musicólogo François Delalande exprimem claramente essa relação dialética.

Para a autora, por exemplo, o advento da notação e da teoria musicais possibilitaram o desenvolvimento da música ocidental tal qual a conhecemos. Segundo ela:

A notação pressupõe o estabelecimento de códigos, de regras e de técnicas (técnica de gravação, por exemplo). O desenvolvimento de teorias pressupõe as análises, as observações, a conceituação, a formalização de regras, assim como, concernindo a acústica, equipamentos de medição acústica e de análise física. $\mathrm{O}$ cálculo e a lógica dispensando quase totalmente - até recentemente - as tecnologias elaboradas, a tecnologia subjacente à evolução da teoria da música é, de fato, aquela relacionada com a própria notação (Heinrich 2003, p. 11). ${ }^{2}$

\footnotetext{
${ }^{1}$ Grisey 2008, p. 246. Tradução nossa. Texto original: “L'essentiel n'est pas d'ajouter à sa pensée une dimension technologique, mais de discerner l'impact que cette dimension peut avoir sur la pensée".

2 Tradução nossa. Texto original: “(...) le développement de la musique occidentale n'a pu être ce qu'il est qu'à travers le rôle fondamental de la notation et de la théorie de la musique (...). La notation a supposé la mise en place de codes, de règles et de techniques (technique de la gravure, par exemple). Le développement des théories suppose des analyses, des observations, la conceptualisation, la formalisation de règles, ainsi que, concernant l'acoustique, des appareillages de mesure et d'analyse physique. Le calcul et la logique se passant presque totalement - jusqu'à récemment - de technologies élaborées, la technologie sous-jacente à l'évolution de la théorie musicale n'est en fait que celle liée à la notation elle-même".
} 
MUSICA THEORICA Revista da Associação Brasileira de Teoria e Análise Musical 2018, v. 3, n. 2, p. 125-134 - Journal of the Brazilian Society for Music Theory and Analysis@ TeMA 2018 - ISSN 2525-5541

François Delalande, por sua vez, nos aponta a influência que as mudanças tecnológicas têm na escrita musical:

Antes do século XII, a música parecia essencialmente oral e transcrita após sua realização. Mas se pularmos para o século XIV, encontramos peças cujos métodos de escrita são, no sentido literal, inimagináveis sem a ajuda do papel (pensamos em retrogradações, dentre os quais o rondó de Guillaume de Machaut, Ma Fin est mon commencement é o exemplo canônico). Evidentemente, elas foram compostas diretamente nesse suporte (Delalande 2003, p. 541). ${ }^{3}$

Encontramos reflexões similares, embora sob ângulos bem diferentes, no discurso de pesquisadores pertencentes a disciplinas absolutamente diversas no campo das ciências sociais e humanas. Entre eles, podemos citar o compositor e filósofo Hugues Dufourt (2004), o filósofo Pierre Lévy (1990), o filósofo e sociólogo Herbert Marshall McLuhan (1968), o antropólogo Jack Goody (1979), etc.

Em linhas gerais, há sempre três fases evocadas concernindo a racionalidade humana: a invenção da escrita, que se sobrepõe à oralidade e introduz uma nova era cognitiva, e a invenção das tecnologias analógicas e digitais que, da mesma forma, sobrepõem-se à escrita.

François Delalande $(1996,2003)$ define como sendo três o número de grandes "paradigmas tecnológicos"4 existentes na história da música:

1. A tradição oral;

2. A escrita;

3. A eletroacústica;

\footnotetext{
3 Tradução nossa. Texto original: "Avant le XIIe siècle, la musique semble essentiellement orale et transcrite après coup. Mais si l'on saute au XIVe siècle, on trouve des pièces dont les procédés d'écriture sont, au sens propre, inimaginables sans l'aide du papier (on pense aux rétrogradations dont le rondeau de Guillaume de Machaut, Ma Fin est mon commencement, est l'exemple canonique). Manifestement, elles ont été composées directement sur ce support".

${ }^{4}$ Segundo Delalande (2003, loc. Cit.), esses paradigmas são circunscritos por: “(...) um conjunto coerente de técnicas, eventualmente de materiais, mas também de práticas sociais, de circuitos de difusão, de formação, tudo isso constituindo as condições do pensamento musical em si e da emergência de 'linguagens' específicas". Tradução nossa. Texto original: “(...) un ensemble cohérent de techniques, éventuellement de matériels, mais aussi de pratiques sociales, de circuits de diffusion, de formation, tout ceci constituant les conditions de la pensée musicale elle-même et de l'émergence de 'langages' spécifiques".
} 
Pierre Lévy (1990), por sua vez, ao tratar da problemática por ele denominada "os três tempos do espírito", propõe a seguinte classificação:

1. A oralidade primária;

2. A escrita;

3. A informática.

Considerando as duas listas, observamos a divergência de proposições quanto à definição da terceira grande etapa tecnológica do intelecto humano. Assim, ao contrário de François Delalande, para Pierre Lévy, a terceira grande revolução cognitiva não corresponde ao advento de equipamentos eléctricos e eletrônicos analógicos (que tornaram possível a invenção da música eletroacústica), mas apenas à tecnologia digital dos computadores. Podemos, a partir dessa constatação, propor dois questionamentos importantes: 1) Por quê há essa divergência entre os dois pesquisadores e 2) o que leva a essa diferença de posições?

Para respondermos a tais perguntas, foquemo-nos no caso específico da música ocidental. Nesse campo, essa incompatibilidade de posição com a lista proposta por Pierre Lévy pode ser compreendida na medida em que é a partir da utilização da tecnologia elétrica ou eletrônica analógica que podemos assistir à consolidação da exploração do ruído como material composicional. Essa conquista se mostra crucial para a história da música contemporânea ocidental porque é através dela que a compreensão do fenômeno sonoro enquanto tal, em detrimento da antiga concepção segundo uma formalização simbólica ${ }^{5}$ da música, se concretiza. Como consequência, temos, sobretudo após as experiências oferecidas pelas músicas concreta ${ }^{6}$ e eletrônica (e posteriormente eletroacústica), uma mudança da unidade básica conceitual da música, que passa da noção de nota à de frequência. ${ }^{7}$

\footnotetext{
${ }^{5}$ A nota, forma de pensamento que se torna possível através da escrita no papel - que permite a decomposição do fenômeno sonoro em parâmetros abstratos e distintos, normalmente simbolizados por: altura, duração, ritmo e timbre.

${ }^{6}$ Entre elas, a experiência do "sillon fermé", ou sulco fechado, nos discos de vinil, que permite, através da repetição contínua de um ruído, não ouví-lo mais de maneira denotativa, mas por suas características morfológicas intrínsecas.

7 Essa afirmação será explicada pouco à frente nesse mesmo artigo, mas uma explicação mais detalhada desse fenômeno pode ser encontrada na Tese de Doutorado: Catanzaro 2013.
} 
MUSICA THEORICA Revista da Associação Brasileira de Teoria e Análise Musical 2018, v. 3, n. 2, p. 125-134 - Journal of the Brazilian Society for Music Theory and Analysis@ TeMA 2018 - ISSN 2525-5541

Apesar de já prenunciada por compositores como Gialcinto Scelsi - que, mesmo sem o recurso de instrumentos eletroeletrônicos afirmaria que em um só som era possível de se ouvir movimentos concêntricos e divergentes, contrapontos (ou deslocamentos de timbres diferentes), e harmônicos que causam efeitos muito diferentes e que penetram no centro do som (2006, p. 6284) -, esse, na exploração musical, não pôde ser encarado como uma matéria plástica até o fim da década de 1940. Isso porque, até esse momento, a ciência e a tecnologia que puderam intervir no pensamento musical eram baseadas apenas nos estudos acústicos conhecidos à época (através da pesquisa de físicos como Hermann von Helmholtz, Henri Bouass, John William Strutt, Rayleigh Dayton, C. Miller, Harvey Fletcher, etc.) e da tecnologia de instrumentos eletroeletrônicos (desenvolvidos por inventores como Taddheus Cahill e Léon Theremin entre outros) utilizados, majoritariamente, de forma tradicional. ${ }^{8}$

No fim da década de 1940, no entanto, surgiram os meios para se explorar o ruído de maneira mais sistemática nas rádios governamentais (primeiramente na França e na Alemanha, e posteriormente em outras partes do mundo como Itália, Japão e Estados Unidos). Surgiriam assim as estéticas das músicas concreta e eletrônica, e seus posteriores desenvolvimentos com a música eletroacústica.

Delas, podemos conservar algumas ideias importantes, que nos permitem entender por quê François Delalande coloca a revolução eletroacústica como o terceiro paradigma tecnológico da história da música:

1. A de escuta reduzida, elaborada meticulosamente no Tratado dos Objetos Musicais de Pierre Schaeffer (1966), que permitiu a instauração de um estudo tipo-morfológico dos sons e sua proposição de uma nova concepção do solfejo, centrado sobre o objeto musical (massa, timbre harmônico, grão, dinâmica, allure, perfil melódico e perfil de massa) e não mais sobre a dualidade nota/ritmo.

2. A da síntese sonora, que abre a possibilidade de forjar a constituição interna dos sons em si, alargando os limites desse controle ao átomo do som (até à possibilidade de serializar e compor, ou escrever, a própria estrutura do timbre). Esse marco

\footnotetext{
${ }^{8}$ Essas pesquisas, não obstante, auxiliaram na criação de teorias composicionais muito frutíferas e que foram objeto de publicações de compositores como Ferruccio Busoni (1907), Henry Cowell (1930), e de escritores como Ezra Pound (1927).
} 
leva à incorporação, como defendeu Stockhausen (2000, p. 88-111), de quatro grandes contribuições à composição contemporânea:

a) A unificação da estrutura temporal (em um contínuo entre ritmo e altura, tal qual explicado no artigo "A unidade do tempo musical") (1959, p. 10-40);

b) A decomposição do som em seus elementos constitutivos estruturais;

c) A composição espacial do som (que implica não somente a espacialização tridimensional durante a difusão da obra, como a composição de diversos planos sonoros com distâncias distintas);

d) E, finalmente, a equidade entre o som com alturas definidas (tones) e o ruído (noise), em um contínuo sonoro definido pelo grau de periodicidade do conteúdo espectral do som (os mais aperiódicos sendo os mais ruidosos).

Karlheinz Stockhausen (2000) diria ainda, nesse mesmo texto, que a nova tecnologia que permitiu o advento da música eletrônica, transformando o método de composição, transformou o homem, e, consequentemente, seu pensamento (p. 88). Parece-nos cabível englobar aqui, igualmente, as conquistas da música concreta francesa. Mesmo se considerarmos as diferenças intrínsecas a essas duas correntes, uma vendo o objeto desde seu exterior (ruído) e a outra tentando sintetizá-lo e compô-lo a partir de sua estrutura mínima (senoide), é possível dizer que, ao separar o som da sua causalidade e considerá-lo apenas por suas qualidades sonoras específicas, para o primeiro, ou ao concebê-lo como síntese, para a segunda, ambas liberaram, imediatamente, o som de suas restrições simbólicas: passamos do campo das notas ao campo da frequência. $\mathrm{O}$ som, a partir de então, não é apenas parte da estrutura composicional. Ele carrega dentro de si uma força estruturante.

Aos poucos, esse imaginário sonoro transborda o universo da música eletroacústica, sendo transposto para a música vocal e instrumental desde a década de 1950. Nascem, dessa forma, novas estratégias de escrita, a fim de aproximar as sonoridades instrumentais e vocais àquelas eletroacústicas, aumentando significativamente o repertório das denominadas "técnicas expandidas" para vozes e instrumentos.

A partir da década de 1960 e especialmente no início de 1970, as conquistas de uma composição baseada no conceito de frequência se multiplicam na música 
MUSICA THEORICA Revista da Associação Brasileira de Teoria e Análise Musical 2018, v. 3, n. 2, p. 125-134 - Journal of the Brazilian Society for Music Theory and Analysis@ TeMA 2018 - ISSN 2525-5541

instrumental e vocal. O timbre é incorporado como célula geradora da obra (através de técnicas expandidas) em diferentes tipos de correntes instrumentais e vocais. A tecnologia da escrita em papel sofre várias fraturas: sinais de todos os tipos são criados para gravar sons que estão além da notação convencional.

Opera-se, durante esse período, num universo entrecruzado por uma música invadida pela esfera do som e ainda notada (e mais ou menos fortemente, pensada) de acordo com estratégias referentes à esfera da nota, mas que incorporam diversas técnicas que provêm dos estúdios: "mixagens", "filtragens", efeitos de "espacialização ", "montagens", “colagens", "sínteses", etc.9

É importante atentarmos para o fato, no entanto, que até o advento da tecnologia digital, as abordagens supracitadas permaneceram ligadas a conceitos de acústica, física e psicofisiologia clássicas. Dessa forma, a escrita instigada por tal tecnologia ainda investiga o timbre a partir de seu exterior, macroscopicamente. Isso ocorre porque esse tipo de abordagem se baseia em uma tecnologia (a eletroacústica) que não permite, por suas ferramentas, reproduzir o perfil dinâmico do som:10 "o tratamento analógico do timbre se limita à filtragem, à modificação do ataque, àquela da curva dinâmica, à reverberação artificial" (Dufourt 2007, p. 5). ${ }^{11}$ Em outras palavras, examinando o som de forma macroscópica, a estrutura física desse resta, por consequência, mal compreendida, fornecendo "indicações frequentemente vagas, flutuantes, ou de categorias contestáveis, e lhe falta[ndo] a confirmação da síntese" (Risset 1994, p. 96). ${ }^{12}$

Com o aparecimento da informática musical, essa situação é finalmente superada. A macroanálise cede lugar à microanálise do fenômeno sonoro. Com essa mudança de escala, vê-se emergir a consciência de que "o som é um complexo dinâmico cuja unidade não é a de um conjunto de peças, mas a de um

\footnotetext{
${ }_{9}$ Como, por exemplo, a realizada por György Ligeti em Lux Æterna sintetizando vocalmente o espectro de um sino.

10 Suas flutuações, transitórios, etc.

${ }^{11}$ Tradução nossa. Texto original: "Le traitement analogique du timbre se limite au filtrage, à la modification de l'attaque, à celle de la courbe dynamique, à la réverbération artificielle".

${ }_{12}$ Nesse trecho, Risset discorre a respeito da música concreta. Tradução nossa. Texto original: “[...] ce nouveau solfège apparaît pour bien d'autres comme inopérant: développé à partir d'écoutes de transformations de sons complexes dont la structure physique est mal connue, il donne des indications souvent vagues, flottantes, ou des catégories contestables, et il lui manque la confirmation de la synthèse".
} 
processo determinado" (idem). A pesquisa subjacente a essa conquista tecnológica, desde então, tem como objetivo "ampliar até à microestrutura do som o papel da notação e os poderes da escrita" (ibidem).

Mesmo que esse desejo já fosse claramente exprimido desde a música eletrônica na década de 1950, a tecnologia digital abre as portas a uma exploração mais consequente do que a que pôde ser realizada pelos compositores que trabalharam com tecnologias analógicas, pelo fato de que "a codificação digital dos sons permite processar materiais e estruturas de modo similar e de considerar verdadeiramente uma sintaxe do som" (Risset 2006, p. 10).13

Jean-Claude Risset (1990) exprime a questão de forma contundente:

A criação eletroacústica do som o faz escapar das constrições mecânicas: mas é somente com os computadores que se pode produzir ou processar o som seguindo modalidades extremamente diversas, de modo sempre preciso e reprodutível. O som não é mais o traço acústico de objetos materiais, ele pode ser programado, construído ou moldado à vontade em vistas de um resultado auditivo (Risset 1990, p. 1). $\cdot^{14}$

A codificação binária torna-se, assim, o meio pelo qual realiza-se a desmaterialização dos fenômenos sensíveis, criando uma nova isomorfia entre, no caso da música, som e número. É essa nova possibilidade de simulação, de análise por síntese, permitida pela máquina, que permite que um novo simbolismo emerja: aquele extraído do cerne da própria matéria.

Se voltarmos às duas listas que propusemos inicialmente, podemos compreender, agora, a razão que leva o terceiro paradigma a não ser idêntico a ambas. De fato, presenciamos duas revoluções tecnológicas distintas no decorrer do século XX: a revolução da transdução - que permitiu o advento da música eletroacústica - e a revolução da codificação - que permitiu o advento da música computacional. Todavia, como já mostrado anteriormente, apenas a tecnologia

\footnotetext{
${ }^{13}$ Tradução nossa. Texto original: “[...] le codage numérique des sons permet de traiter matériaux et structures de façon similaire et d'envisager une véritable syntaxe du sonore".

${ }_{14}$ Tradução nossa. Texto original: “La création électroacoustique du son le fait échapper aux contraintes mécaniques : mais ce n'est qu'avec l'informatique qu'on peut produire ou traiter le son suivant des modalités extrêmement diverses, de façon toujours précise et reproductible. Le son n'est plus la trace acoustique d'objets matériels, il peut être programmé, construit ou infléchi à volonté en vue d'un résultat auditif. D'autre part la notation musicale a suggéré certaines transformations compositionnelles, comme le renversement ou la récurrence : la puissance de manipulation logique qu'offre l'informatique est à même de démultiplier le rôle de la notation structurale et de proposer de nouveaux modes de composition".
} 
MUSICA THEORICA Revista da Associação Brasileira de Teoria e Análise Musical 2018, v. 3, n. 2, p. 125-134 - Journal of the Brazilian Society for Music Theory and Analysis@ TeMA 2018 - ISSN 2525-5541

digital oferece de a possibilidade desmaterializar o fenômeno a fim de compreendê-lo através de uma microanálise, e de simulá-lo, ou criá-lo, através de uma micro-escritura, sem nenhuma constrição material.

\section{Referências}

1. Busoni, Ferruccio. 1907. Esquisse d'une nouvelle esthétique musicale. In Pierre Michel (org.). Ferrucio Busoni : L'esthétique musicale, Textos traduzidos do alemão para o francês por Daniel Dollé e Paul Masotta. Paris: Minerve, 1990, p. 19-56.

2. Catanzaro, Tatiana. 2013. La Musique Spectrale face aux apports technoscientifiques. Paris: Université de Paris IV - Sorbonne.

3. Catanzaro, Tatiana. 2018. Transformações na linguagem musical contemporânea instrumental e vocal sob a influência da música eletroacústica entre as décadas de 1950-70. Rio de Janeiro: 7Letras.

4. Cowell, Henry. 1930. New musical resources; with notes and an accompanying essay by David Nicholls. Melbourne/Australia: Cambridge University Press, 1996.

5. Delalande, François. 1996. La musique électroacoustique, coupure et continuité. Musurgia: La création musicale et l'analyse aujourd'hui, vol. 3, n. 3, p. 36-55.

6. 2003. Le paradigme électroacoustique. In Jean-Jacques Nattiez (ed.). Musiques: Une encyclopédie pour le XXIe siècle, vol. 1: Musiques du XXe siècle. Paris: Actes Sud/Cité de la musique, p. 533-557.

7. Dufourt, Hugues. 2004. Temps et espaces de l'écriture musicale: de la notation à l'ordinateur. In Nicolas Donin e Bernard Stiegler (ed.). Cahiers de Médiologie/Ircam n. 18: Révolutions Industrielles de la Musique. Fayard: Novembro, p. 67-74.

8. Dufourt, Hugues. 2007. De la dimension productive de l'intensité et du timbre et leur intégration au système des 'éléments porteurs de forme', Actes $d u$ Colloque international: Composer au XXIe siècle - Processus et Philosophies. Colóquio do Observatoire International de la Création Musicale (OICM). Montréal: Faculdade de Música, Universidade de Montréal, março, p. 107118.

9. Goody, Jack. 1979. La Raison graphique. La domestication de la pensée sauvage. Traduzido do inglês para o francês e apresentado por Jean Bazin e Alban 
Bensa. Paris: Éditions de Minuit, 1979. Título original: The domestication of the savage mind (Cambridge: CUP, 1977).

10. Grisey, Gérard. 2008. Le compositeur présenté par son éditeur, entretien avec Ivanka Stoüanova. In Guy Lelong (ed.). Gérard Grisey: Écrits ou l'invention de la musique spectrale, com a colaboração de Anne-Marie Réby. Paris: Éditions MF, Coleção "Répercussions", p. 243-248.

11. Heinrich, Marie-Noeille. 2003. Création musicale et technologies nouvelles: mutation des instruments et des relations. Paris: L'Harmattan.

12. Lévy, Pierre. 1990. Les technologies de l'intelligence: L'avenir de la pensée à l'ère informatique. Paris: La Découverte.

13. McLuhan, Herbert Marshall. 1968. Pour Comprendre les Médias: Les prolongements technologiques de l'homme. Traduzido do inglês para o francês por J. Paré. Montréal: Éditions HMH. Título original: Understanding Media: The Extensions of Man (New York: McGraw-Hill, 1964).

14. Pound, Ezra. 1927. Antheil: with a treatise on harmony with supplementary notes by Ezra Pound. Chicago: Pascal Covici.

15. Risset, Jean-Claude. 1990. Composer le son: expérience avec l'ordinateur, 1964-1989. Contrechamps: Musiques Électroniques, vol. 11. Paris: L'Âge de l'Homme, p. 107-126.

16. Risset, Jean-Claude. 1994. Quelques aspects du timbre dans la musique contemporaine. In A. Zenatti (ed.). Psychologie de la musique. Paris: Presses Universitaires de France, p. 87-114.

17. Risset, Jean-Claude. 2006. Préface. Horacio Vaggione: vers une syntaxe du sonore. In Makis Solomos (ed.), Espaces composables: essais sur la musique et la pensée musicale d'Horacio Vaggione. Paris: L'Harmattan, Coleção “Esthétiques", p. 5-18.

18. Scelsi, Giacinto, Mallet, Franck, et al. 2006. Conversation avec Giacinto Scelsi. In Sharon Kanach (ed.), Les anges sont ailleurs... Arles: Actes Sud, p. 62-84.

19. Schaeffer, Pierre. 1996. Traité des objets musicaux. Paris: Seuil.

20. Stockhausen, Karlheinz. 1959. ....How time passes.... Tradução para o inglês realizada por Cornelius Cardew, Die Reihe, n. 3, p. 10-40. Publicado originalmente sob o título ...Wie die Zeit vergeht..., Die Reihe, n. 3, 1957.

21. Stockhausen, Karlheinz. 2000. Four criteria of electronic music. In Robin Maconie (ed.), Stockhausen on music: Lectures and Interviews compiled by Robin Maconie. Londres: Marion Boyars Publishers Ltd, p. 88-111. 